\title{
Puntos de vista y esquemas argumentales en el discurso de Francisco Huenchumilla en el marco del conflicto Estado-pueblo mapuche
}

\author{
Standpoints and Argument Schemes in the Speech \\ of Francisco Huenchumilla About the So-Called \\ Mapuche Conflict
}

\author{
CECILIA QUINTRILEO LLANCAO \\ Universidad Austral de Chile, Facultad de Filosofía y Humanidades. \\ Correo electrónico: cecilia.quintrileo@uach.cl
}

Hoy en día, el movimiento mapuche constituye un fenómeno de enorme complejidad e impacto social, de manera que su presencia en la sociedad y política chilena resulta insoslayable. Desde un enfoque de análisis del discurso argumentativo, este trabajo se centra en el discurso de Francisco Huenchumilla, político de amplia trayectoria en materia de políticas públicas e indígenas en los últimos decenios. La relevancia de su discurso obedece al posicionamiento discursivo de Huenchumilla en diferentes medios y a una serie de controversias enfrentadas durante su gestión como intendente de la región Araucanía en el marco del denominado conflicto mapuche. El estudio es de carácter cualitativo y su objetivo es analizar los puntos de vista y esquemas argumentales de Huenchumilla respecto del denominado 'conflicto mapuche'. El corpus se conforma de un conjunto de secuencias discursivas emitidas por Francisco Huenchumilla en programas televisivos chilenos. El marco teórico-metodológico se nutre de la teoría pragma-dialéctica y contempla análisis argumentativo y pragmático del discurso. Los resultados del análisis permiten concluir que Francisco Huenchumilla utiliza maniobras estratégicas específicas de posicionamiento de la causa mapuche en los medios, a través de esquemas argumentales específicos, conjugando 'eficacia' y 'razonabilidad'.

Palabras claves: conflicto mapuche, discurso argumentativo, puntos de vista, esquemas argumentales, Francisco Huenchumilla.

Nowadays, the Mapuche movement is a phenomenon of enormous complexity and social impact, so that its presence in Chilean society and politics is unavoidable. In this article, the so-called conflict between the State of Chile and the Mapuche people is seen from an argumentative discourse analysis approach, focusing on the discourse of Francisco Huenchumilla, a politician with broad experience in the national scenario in public and indigenous policies in recent decades. The relevance of his discourse is also due to the discursive positioning of Huenchumilla in different media, and to a series 
of controversies faced during his tenure as Intendant of the Araucanía region within the so-called Mapuche conflict. The study is qualitative and aims to analyze the standpoint and argument schemes in the speech of Francisco Huenchumilla about the so-called Mapuche conflict. The corpus is made up of a set of discursive sequences, broadcast by Francisco Huenchumilla in television programs. The theoretical-methodological framework is nourished by the pragmatic-dialectic theory and contemplates argumentative and pragmatic analysis of the discourse. The results of the analysis allow us to conclude that Francisco Huenchumilla uses specific maneuvers to position the Mapuche cause in the media, through specific argument schemes, combining 'efficacy' and 'reasonableness'.

Key words: Mapuche conflict, argumentative speech, standpoint, argument schemes, Francisco Huenchumilla.

\section{INTRODUCCIÓN}

Un aspecto relevante de la protesta indígena en Chile se visualiza claramente en torno a demandas políticas sobre derechos ancestrales y una serie de acontecimientos que se conocen mediáticamente como el denominado 'conflicto mapuche' (Bengoa 2012, Mella 2007). ${ }^{1}$ El debate se ha dado en el marco de un escenario complejo, no exento de conflictos sociales y jurídicos. Ante los derechos internacionalmente creados, por ejemplo, Chile es conocido por las serias dificultades socio-políticas que ha debido enfrentar en relación con el resguardo de los DD.HH. de los pueblos indígenas, pues sus acciones, a menudo, han sido reprimidas por el Estado (Durán 2007, Bengoa 2012).

Desde una mirada dialéctica del fenómeno, analistas de la situación actual de los pueblos indígenas han señalado la responsabilidad que le cabe al Estado en la agudización e irresolución de este conflicto (Pinto 2015), contribuyendo a un poderoso y emergente movimiento social que asume nuevas formas de organización, en algunos casos, dotadas de un programa político de gran radicalidad (Goicovic 2015). En el estado de la cuestión, la ocupación del territorio mapuche por parte del Estado desde mediados del siglo XIX constituye uno de los principales factores del conflicto, además de la fuerte expansión del rubro forestal, que tiene control sobre los territorios que las comunidades reclaman para sí, provocando profundos cambios en el mercado de la tierra, del ecosistema ambiental y sociocultural, resultando nocivos para los pequeños agricultores mapuche (Pinto 2015); en otros casos, el Estado de Chile ha dado luz verde a la construcción de represas hidroeléctricas en territorio mapuche, poniendo la cuestión del progreso por sobre los derechos de los pueblos indígenas, en general, y mapuche, en particular (Pairicán 2015).

\footnotetext{
${ }^{1}$ El inicio de las acciones reivindicativas del pueblo mapuche en el sur del país serán conocidas mediáticamente como el denominado "conflicto mapuche". La atención de los medios de comunicación y de la prensa, en particular, comenzará a partir del $1^{\circ}$ de diciembre de 1997 cuando un grupo de mapuches intercepta un convoy de camiones de la empresa forestal Bosques Arauco y quema tres de esos vehículos. Dos días más tarde de este hecho, comenzará la ofensiva comunicacional de los principales periódicos: El Mercurio de Santiago y La Tercera (Amolef, 2017).
} 
Hoy en día, el movimiento mapuche constituye un fenómeno de enorme complejidad e impacto social, de manera que su presencia en la sociedad y política chilena resulta insoslayable (Bengoa 2012). El conflicto es percibido por la opinión pública como una movilización creciente y progresiva que se extiende a lo largo de toda la Araucanía (actuales VIII, IX y X regiones de Chile). En la formación de esta visión, los medios de comunicación han tenido un papel trascendental (Vergara, Gunderman y Foester 2013), no siendo desconocido el actual fenómeno de criminalización que se da en torno a la protesta indígena en el país (Mella 2007).

Históricamente, desde el año 1992, los Gobiernos de turno han manifestado variadas intensiones de diálogo (Vergara, Gunderman y Foester 2013); pero, a toda vista, desde una perspectiva de los procesos discursivos-argumentativos, propios de los regímenes democráticos (Perelman y Olbrechts-Tyteca 2009), los cursos de acción no siempre han sido los más acertados, invocándose estrategias en materia de seguridad, que lejos de resolver los conflictos sociales, los han aumentado, debido a la aplicación de normativas de excepción, como la Ley de Seguridad Interior del Estado y Ley Antiterrorista (Yañez, 2007). En años recientes, en el programa del segundo gobierno de Michelle Bachelet (2014- 2017) en materia de pueblos indígenas se asumen varias medidas, ${ }^{2}$ haciendo suponer una nueva forma de relación o interacción del Estado con los pueblos indígenas. ${ }^{3}$ Un punto destacado en el Gobierno de Bachelet es el nombramiento de Francisco Huenchumilla como intendente de la región de La Araucanía, lugar donde la protesta indígena se visibiliza mayormente. Durante su gestión, Francisco Huenchumilla despliega una política de acercamiento a las comunidades indígenas de su región, recibiendo, por ello, duras críticas de diferentes sectores políticos, tanto de la oposición como del oficialismo. Sin duda, su discurso marca un hito político e histórico, por sus controversiales declaraciones, pidiendo 'perdón' al pueblo mapuche en nombre del Estado por los abusos cometidos históricamente, y otras expresiones como 'quemarse a lo bonzo', para lograr la paz en la Araucanía (Cayuqueo 2015), además de una serie de acciones como su visita a dirigentes encarcelados en el marco del caso Luchsinger. ${ }^{4}$

\footnotetext{
${ }^{2}$ Estas medidas forman parte de los 50 compromisos para los primeros 100 días de Gobierno de M. Bachelet. En su Programa de Gobierno, la Presidenta Bachelet destaca la importancia de los asuntos indígenas, a través de compromisos políticos explícitos, como la creación de un Ministerio de Asuntos Indígenas, encargado de "colaborar con el Presidente de la República en la definición de una política indígena para Chile", además del envío al Congreso de un proyecto de ley, para la creación de un Consejo de Pueblos Indígenas, como una institución autónoma y representativa de los diversos pueblos que existen en Chile" para la atención de requerimientos en materia de educación, salud, tierras y desarrollo productivo.

${ }^{3}$ http://michellebachelet.cl/pdf/50medidasMB.pdf.

${ }^{4}$ Caso judicial chileno en torno al atentado incendiario llevado con resultado de muerte del matrimonio conformado por el empresario Werner Luchsinger y Vivianne Mackay. Hasta hoy, el único imputado en este caso es Celestino Córdoba, aun cuando se han procesado a una serie de personas, invocándose normativas de excepción, como la Ley Antiterrorista. Los hechos han sido ampliamente difundidos por la prensa chilena, con opiniones divididas en torno al fallo de los Tribunales chilenos.
} 
En este trabajo, se estudia el discurso político de Francisco Huenchumilla desde un enfoque de análisis del discurso argumentativo con el objetivo de analizar sus puntos de vista y esquemas argumentales respecto del denominado 'conflicto mapuche'. El marco conceptual y metodológico del estudio se nutre principalmente del campo de la argumentación, particularmente desde el enfoque pragma-dialéctico (Van Eemeren y Grootendorst 2002 y 2004, Van Eemeren 2012). El trabajo se estructura en dos apartados: el primero presenta el marco conceptual y metodológico, el segundo, se centra en el análisis y discusión de los resultados, para finalmente exponer las conclusiones.

\section{MaRCo CONCEPTUAL Y METODOLóGico}

El estudio de la práctica argumentativa del lenguaje ha sido objeto de especial atención en campos como la filosofía, el derecho, la publicidad, la teoría política, la lingüística, entre otros (Martínez 2005, Marafioti y Santibánez 2010, Van Eemeren 2012). Uno de los puntos clave en su reconocimiento y valoración es el desarrollo de sociedades democráticas cada vez más intercomunicadas en el plano político, informativo, económico, etc., cobrando especial relevancia en relación con el papel que puede desempeñar en el discernimiento de los obstáculos -intereses e ideologías- involucrados en la toma de decisiones en la vida pública. Los miembros y partidarios de la democracia manifiestan clara conciencia sobre el valor que tiene la argumentación, por una parte, para la defensa o refutación de ideas; por otra, para develar y/o criticar un tipo de argumentación subyacente, relacionada con conflictos de intereses (Campz y Dolz 1995).

En este trabajo, utilizamos el concepto de argumentación desde un punto de vista discursivo, en tanto evento comunicacional e interactivo (Van Eemeren 2010). En las líneas que siguen se describe la teoría pragma-dialéctica, marco conceptual del estudio, para centrarnos posteriormente en el marco metodológico.

\subsection{La teoría pragmadialéctica}

La perspectiva pragmadialéctica se alinea con las aproximaciones pragmáticas del discurso (Austin 1982, Searle1969, Grice 1975), tomando como punto de partida el diálogo con participantes definidos como tales, aspecto que constituye una innovación considerable en el estudio de la argumentación (Gille 2001). Desde esta óptica, la argumentación se define como una actividad verbal, social y racional, destinada a convencer a alguien sobre la aceptabilidad de un punto de vista, mediante una serie de argumentos que permiten la justificación o refutación de dicho punto de vista (Van Eemeren y Grootendorst 2002, 2004, Van Eemeren 2012).

Este modelo teórico conjuga tres dimensiones analíticas: dialéctica, pragmática y retórica. La dimensión dialéctica sitúa la argumentación entre dos partes, comprometidas en un procedimiento de discusión que les permite alcanzar sus metas argumentativas 
razonablemente. La dimensión pragmática define la argumentación como un macroacto de habla, conformado por la sucesión de una serie de actos de habla elementales, que cumplen una función relevante en el proceso de resolución de una disputa. La dimensión retórica se centra en los aspectos retóricos, en términos de las maniobras estratégicas o argumentales, que abarcan diferentes mecanismos utilizados por los participantes de una discusión con la finalidad de ser eficaces (Van Eemeren y Houtlosser 2007).

La argumentación es la acción comunicativa que surge cuando existe una diferencia de opinión, tanto en el ámbito cotidiano o familiar, como en contextos más institucionalizados, como, por ejemplo, el debate político, presidencial o parlamentario, o los alegatos de los abogados en los tribunales de justicia. En cualquier caso, los participantes del acto de argumentación pueden tener diferentes propósitos o metas, cuya consecución depende de dos principios fundamentales: razonabilidad y eficacia.

Razonabilidad. Si la meta es resolver diferencias de opinión de una manera razonable, el orador considera como principio vector de su discurso la razonabilidad, donde la idea de lo "razonable" se refiere a hacer uso de la razón de forma apropiada, de acuerdo a la situación; de esta manera, un procedimiento argumentativo es razonable si está en concordancia con normas de interacción tácitas, como el principio de cooperación y las máximas conversacionales, en cuanto a calidad, cantidad, modalidad y pertinencia (Grice 1975) de los puntos de vista y argumentos presentados en una discusión. Asimismo, la razonabilidad se define en función de reglas pragma-dialécticas, las cuales cumplen una función específica en relación con la resolución de una diferencia de opinión y, al mismo tiempo, cada una de ellas permite identificar, de modo preciso, qué falacias se deben controlar en una discusión.

Eficacia. Este principio constituye la dimensión perlocutiva o interactiva del acto de argumentar, y es relativo a los efectos que busca provocar el orador con su discurso, mediante sus argumentos, poniendo en juego una serie estrategias para influir en una audiencia específica. Van Eemeren (2012) agrupa las distintas estrategias argumentativas en el concepto de maniobra estratégica o argumentativa, las que se definen como "medios para que los argumentadores alcancen sus objetivos retóricos, respetando al mismo tiempo los requisitos para resolver las diferencias de opinión de una forma razonable" (Van Eemeren 2012).

La maniobra estratégica concierne tres aspectos fundamentales: a) La selección de tópicos o esquemas argumentales, b) La orientación hacia la audiencia y c) Los mecanismos de estilo o figuras retóricas. Cada uno de estos aspectos se integra en el análisis del discurso; el primer aspecto, el análisis de los tópicos o esquemas tiene como propósito conocer los tipos de argumentos que refuerzan los puntos de vista, aludiendo a la fuerza argumental del discurso, la cual se refiere al grado de seguridad con el que se adopta un punto de vista (Van Eemeren, Grootendorts y Snoek Henkemans 2006); el segundo aspecto ayuda a conocer la interpelación de diferentes entes, individuales o colectivos, de parte del orador en su discurso por medio de ciertas expresiones que permiten captar su atención, determinando el alcance de los argumentos, el cual apunta a la cantidad de individuos sobre los cuales se aplica la proposición argumentativa; y el tercer aspecto, permite explorar los mecanismos discursivos o estilísticos, utilizados para reforzar los puntos de vista o el conjunto de las argumentaciones. Los 
tópicos potenciales, referentes a los esquemas argumentativos; las demandas de la audiencia, a los puntos de partida que la audiencia pueda aceptar; y los mecanismos presentacionales, a la forma en que se presentan los movimientos argumentativos, son aspectos que funcionan de forma interdependiente y coordinada en cada movimiento argumental.

Las maniobras argumentativas o estratégicas surgen como un elemento que tiene como objetivo alcanzar un equilibro entre la razonabilidad y la eficacia, donde la razonabilidad se relaciona con la perspectiva dialéctica y la eficacia con la perspectiva retórica de la argumentación. Por tanto, la razonabilidad persigue un objetivo dialéctico: conseguir la resolución de la diferencia de opinión, mientras que la eficacia persigue un objetivo retórico: conseguir la aceptación de la audiencia.

Esquemas argumentales. Los esquemas argumentales representan la organización interna de cada argumentación única, conformando maneras más o menos convencionalizadas de mostrar la transferencia de la aceptabilidad de las premisas al punto de vista. Van Eemeren, Grootendorsts y Snoeck Henkemans (2006) proponen tres categorías de esquemas argumentativos, los cuales representan una estrategia de argumentación diferente: a) argumentación basada en una relación sintomática; b) argumentación basada en una relación causal y c) argumentación basada en una relación de analogía. El primer tipo de argumento, de base causal, se refiere a la relación punto de vista y argumento, adoptado como antecedente, motivo o razones, para justificar la aceptabilidad del punto de vista. Este abarca igualmente el argumento causal-pragmático o argumento por la consecuencia, donde se toma como argumento las potenciales consecuencias (des) favorables de adoptar una determinada línea de conducta (Van Eemeren, Grootendorts y Snoeck Henkemans 2006). El segundo tipo de argumento, de base sintomática, se refiere al uso de argumentos que muestran un signo o cualidad distintiva de lo planteado en el punto de vista. Algunos sub tipos de esta relación son: el argumento por el ejemplo, en el que se presenta un número de casos como representación de algo más general; el argumento por definición, en el que es posible relacionar una razón con un punto de vista utilizando una definición; y el argumento por autoridad, en el que se recurre a una fuente de prestigio o al conocimiento de un experto para establecer una conclusión (Van Eemeren, Grootendorts y Snoeck Henkemans 2006). Por último, en la argumentación por analogía se defiende un punto de vista sobre la base de una suerte de paralelo o semejanza, afirmando que lo que se ha mencionado en el punto de vista tiene un correlato en la experiencia, recurriendo a una comparación o analogía entre dos situaciones o casos. Evidentemente, el análisis de los esquemas argumentales resulta de gran interés para conocer cómo se refuerza la relación argumento-punto de vista, como una dimensión específica de la maniobra estratégica.

\subsection{Marco metodológico}

En este trabajo, se estudia el discurso político de Francisco Huenchumilla y su punto de vista respecto del 'conflicto mapuche' desde un enfoque de análisis del discurso argumentativo. La relevancia de este actor político resulta innegable en el contexto de las 
políticas públicas e indígenas en los últimos decenios, junto con convertirse en el primer intendente de ascendencia mapuche en la región de la Araucanía, posicionando las demandas de este pueblo en distintos medios informativos, y, con ello, enfrentando una serie de controversias durante su gestión en el marco del denominado conflicto mapuche. El marco metodológico se articula desde la teoría pragma-dialéctica, conjugando análisis argumentativo y análisis pragmático del discurso, de acuerdo a Van Eemeren y Grootendorts (2002, 2004); Van Eemeren, Grootendorts y Snoeck Henkemans (2006) y Van Eemeren (2012). El análisis pragmadialéctico de los esquemas argumentales se realiza desde la noción de 'maniobra estratégica (Van Eemeren 2012), y se complementa con la perspectiva retórica de Perelman (1968), el principio de cooperación y las máximas conversacionales (Grice 1975), y las preguntas críticas aplicadas a los esquemas argumentativos, propuestas por Walton (1989). Finalmente, el análisis del contexto pragmático, se complementa con la consideración de aportes de distintos teóricos, que han contribuido a la discusión del 'conflicto mapuche', principalmente desde el campo de las ciencias sociales.

\subsubsection{Objetivo y características del corpus}

El estudio es de carácter cualitativo y tiene por objetivo analizar los puntos de vista y esquemas argumentales en el discurso de Francisco Huenchumilla, en torno al denominado conflicto mapuche. El corpus se conforma de un conjunto de secuencias discursivas, emitidas por Francisco Huenchumilla en distintos programas televisivos, de género entrevista y/o debate político, presentados entre 2014 y 2015, período de gestión del ex intendente Huenchumilla en la región de la Araucanía. Entre estos programas, se encuentran: "Tomás Mosciatti en CNN Chile" (5 junio de 2014); "Mentiras Verdaderas" (19 de junio de 2014) y Tolerancia Cero (30 de agosto de 2015). ${ }^{5}$

\subsubsection{Procedimiento de análisis}

El análisis de los datos se centra en los puntos de vista y esquemas argumentales identificados en distintas secuencias discursivas, emitidas por el ex intendente Francisco Huenchumilla en los programas televisivos estudiados. Los esquemas argumentativos se presentan a través de figuras que representan la estructura lógica del esquema argumental, según tres proposiciones constitutivas: el punto de vista o conclusión (1), el (los) argumento (s) explícito (s) (1.1.) y la premisa implícita reconstruida (1.1'), la cual muestra el tipo de esquema argumentativo utilizado. La representación de los esquemas argumentativos se realiza de acuerdo a los procedimientos analíticos propuestos en Van Eemeren y

\footnotetext{
${ }^{5}$ En cuanto a los programas televisivos, "Tomás Mosciatti en CNN Chile", se emite por CNN-Chile en coproducción con Radio Bío-Bío bajo la conducción de Tomás Mosciatti; "Mentiras Verdaderas" es un programa estelar de entrevista del canal de televisión abierta La Red (http://lared.cl0). A la fecha de este estudio, el conductor del programa es Jean Phillippe Cretton. "Tolerancia Cero es un programa de debate político y entrevista, conducido a la fecha del estudio por Matías del Río y con participación de destacados panelistas.
} 
Grootendorts (2002, 2004); Van Eemeren, Grootendorts y Snoeck Henkemans (2006) y Van Eemeren (2012).

Las unidades de análisis del discurso se segmentaron en secuencias discursivas, presentes en los intercambios pregunta-respuesta, ${ }^{6}$ par adyacente característico del género entrevista (Tusón 1997). En la selección de las secuencias discursivas se ha considerado principalmente el criterio temático, guiado por las preguntas dirigidas a Francisco Huenchumilla en las interacciones televisivas estudiadas, donde se abordan, entre otras temáticas: la gestión del ex intendente en la región de la Araucanía, la controversia desatada a raíz de su visita a uno de los principales procesados en el caso Luchsinger; la cuestionada Ley Antiterrorista y el diagnóstico sociopolítico del conflicto Estado-pueblo mapuche, desde la mirada de Huenchumilla.

\section{ANÁlisis y discusión de los Resultados}

En este apartado se presenta el análisis y discusión de los resultados, centrándose en la descripción de los puntos de vista y esquemas argumentales identificados en el discurso de Francisco Huenchumilla. El análisis de los resultados revela que el ex intendente, en tanto orador, utiliza una variedad de maniobras estratégicas, en torno al conflicto mapuche, en general, y a su gestión política en tanto intendente de la región de la Araucanía, en particular.

Entre las principales maniobras estratégicas identificadas en el análisis del discurso de Huenchumilla se encuentran esquemas argumentales de base sintomática y de relación causal, que pasamos a describir a continuación. En la descripción de cada esquema argumental, se incluye un fragmento discursivo de la interacción estudiada, destacándose -en negrita- la temática planteada en las preguntas del entrevistador y los enunciados argumentativos, puntos de vista y argumentos, esgrimidos por el entrevistado; asimismo, en cada caso, se representa el esquema argumentativo correspondiente mediante una figura de su estructura lógica.

\subsection{Esquema de argumentación basada en una relación sintomática por signos}

En el esquema de argumentación basada en una relación sintomática, o argumento por signos, el argumento encierra una característica de determinada situación, una calidad inherente de cierta personalidad, una prueba empírica, una evidencia. En este tipo de esquema, el razonamiento es de carácter no deductivo, de modo que el orador establece una relación de plausibilidad fáctica entre las premisas y la conclusión o punto de vista ${ }^{7}$,

\footnotetext{
${ }^{6}$ Par adyacente: se refiere a dos emisiones realizadas por hablantes diferentes, con carácter sucesivo, es decir, un hablante expone una primera parte y solo cuando esta es finalizada, el otro hablante emite una segunda parte (Schegloff y Sacks, 1973; Levinson, 1983).

7 Desde la pragmadialéctica, la noción de 'premisas' y 'argumentos' se utilizan indistintamente. La premisa explicita corresponde al argumento explícito o premisa menor, la premisa implícita a la premisa mayor del silogismo aristotélico. Del mismo modo, las nociones de 'conclusión' y 'punto de vista' se utilizan indistintamente.
} 
de tal manera que tiene amplia libertad discursiva para fundamentar sus puntos de vista, estableciendo conjeturas o presunciones sobre premisas ampliamente admitidas por la audiencia construida por el orador (Perelman 1968). En el análisis del discurso de Francisco Huenchumilla, hemos encontrado este tipo de esquema argumentativo en la fundamentación de sus puntos de vista. A continuación, se describe este tipo de esquema en relación con la controversia desatada a raíz de la visita del ex intendente a Celestino Córdoba, principal procesado en el caso Luchsinger.

Tomás Mosciatti: -En los últimos días hubo una polémica, porque usted fue a visitar a Celestino Córdoba, porque estaba en huelga de hambre. ¿Qué es Celestino

\section{Córdoba para usted?}

Francisco Huenchumilla: - Es una persona condenada por la justicia, por los Tribunales, ehh y una persona que cree en su causa, que [es] la reivindicación del mundo mapuche.

Tomás Mosciatti: -¿Ud. cree que está equivocado Celestino?

Francisco Huenchumilla: -Ehh mire yo soy, en primer lugar, soy respetuoso de los fallos de los tribunales, y no los cuestiono.

Tomás Mosciatti: - ¿O sea que usted cree que él es un asesino?

Francisco Huenchumilla: -No, yo digo que hay una sentencia, que lo condenó. Él tuvo su defensa, tuvo sus abogados. El juicio terminó y en la Araucanía en el último tiempo ha habido muchos juicos con condena y con absoluciones. Y por lo tanto, como autoridad y como abogado, yo estoy a lo que determinen los Tribunales. (Francisco Huenchumilla, en Tomás Mosciatti en CNN Chile, 5 junio de 2014)

En la secuencia presentada, se observa que Tomás Mosciatti, conductor del programa televisivo, indaga en la visita realizada por el entonces intendente a Celestino Córdoba, principal procesado por la justicia chilena en el caso Luchsinger-Mackay, hecho cuestionado por ciertos sectores políticos, principalmente de Oposición. En el análisis de la interacción, se observa que las preguntas del entrevistador constituyen una interpelación tácita al ex intendente, a raíz de la visita realizada a Celestino Córdoba: En los últimos días hubo una polémica, porque usted fue a visitar a Celestino Córdoba, porque estaba en huelga de hambre. ¿Qué es Celestino Córdoba para usted? ¿Ud. cree que está equivocado Celestino? El ex intendente responde, sin embargo, mediante una visión acotada de los hechos, evitando juicios o apreciaciones con respecto a la presunta culpabilidad de Celestino Córdoba, lo que se observa en distintas secuencias de su discurso: "[Celestino Córdoba] es una persona condenada por la justicia, por los Tribunales...”, “... Soy respetuoso de los fallos de los Tribunales, no los cuestiono"; "Yo digo que hay una sentencia, que lo condenó". De alguna forma, el ex intendente, comete la falacia de evadir la carga de la prueba y con ello evita dar a conocer a la opinión pública una visión personal sobre los hechos en el connotado caso Luchsinger, sorteando así, posibles cuestionamientos, principalmente desde sectores de la Oposición. El esquema argumentativo utilizado por Huenchumilla se reconstruye en la siguiente figura: 
Fig. 1. Estructura lógica de esquema argumental de base sintomática: argumento por signos

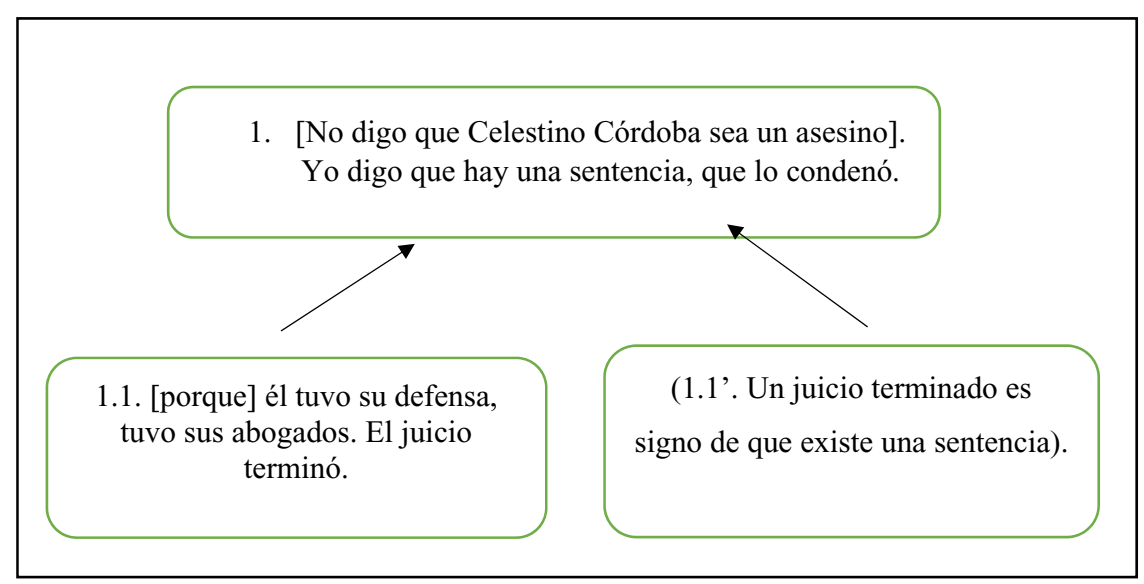

La figura anterior muestra que la relación punto de vista-argumento descansa en un esquema por signos, cuya función específica consiste en entregar una evidencia o prueba en relación a lo afirmado. La naturaleza del esquema argumentativo sintomático puede observarse a partir de la reconstrucción de la premisa implícita (1.1.’), la cual relaciona el argumento con el punto de vista. El contenido proposicional del argumento (1.1.) refiere a aspectos específicos de un proceso judicial como, por ejemplo, los hechos de "tener defensa, tener abogados, juicio concluido", los cuales pueden considerarse una evidencia o signo de una situación concreta, como la sentencia, que ha condenado a Celestino Córdoba, lo que permite sostener el punto de vista planteado (1): "Yo digo que hay una sentencia que lo condenó". Como se observa, la naturaleza del argumento por signos es factual, ya que los hechos que sirven como argumento se pueden constatar a través del seguimiento del proceso y la sentencia del juicio. Desde el punto de vista analítico, por una parte, la alusión a la lógica del proceso jurídico en tanto evidencia empírica muestra la correlación entre lo expuesto en el argumento y la afirmación del punto de vista (1), lo que permite responder a la pregunta crítica sobre ¿cuál es la fuerza de la correlación del signo con el evento significado? Por otra parte, al plantear una segunda pregunta crítica: ¿hay otros eventos que podrían ser fuentes más confiables que tal signo?, podemos señalar que la simple alusión al caso señalado presupone la existencia de sus antecedentes en los tribunales, como el expediente del caso, el proceso y la sentencia. De este modo, si bien el orador podría haber precisado mayores detalles del caso a través de fuentes jurídicas, sería redundante, tratándose de un caso tan emblemático, como el de Luchsinger. Como puede observarse, desde una mirada crítica, la alusión a la lógica del proceso jurídico, principal fuente de co-relación argumento-punto de vista contribuye a la razonabilidad del discurso de Huenchumilla. A su vez, el esquema utilizado resultaría aceptable para la 
consecución de la eficacia discursiva desde una perspectiva empírica, pues el orador ha utilizado adecuadamente la maniobra estratégica del argumento sintomático, legitimando su punto de vista desde su propia experiencia como abogado, desde el campo jurídico, refiriéndose a premisas admitidas y reconocidas ampliamente en el proceso judicial.

Sin embargo, ¿cuán aceptable resulta la argumentación de Huenchumilla? Desde el punto de vista de la maniobra estratégica, encontramos cierta tensión entre el principio de razonabilidad y la eficacia. A simple vista, el orador no responde directamente a algunas preguntas del entrevistador, en particular, aquellas que indagan sobre su visión personal en cuanto a la culpabilidad de Celestino Córdoba en el caso Luchsinger. Más precisamente, Huenchumilla elude la máxima de cantidad (Grice), aportando un mínimo de información, y, a su vez, la máxima de pertinencia, pues evita dar un punto de vista personal acerca de Celestino Córdoba, neutralizando su postura, a través de un cambio de dirección argumentativa, orientada hacia el proceso que dictaminó la sentencia. En otras declaraciones, con respecto a los presuntos culpables en el caso mencionado, el ex intendente recurre a un argumento sintomático por autoridad, evitando cualquier tipo de apreciación personal:

Conductor: ¿Usted cree que el crimen Luchsinger Mackay fue propiciado por mapuches?

F. Huenchumilla: Mire en esa materia yo me atengo a lo que digan los Tribunales de Justicia, no me corresponde a mí juzgar, digamos, hechos delictuales que están siendo investigados o que fueron investigados por los Tribunales. Yo me atengo, lo que se diga en esa materia, a lo que digan los Tribunales. Además, por mi formación de abogado, yo no puedo andar condenando ni absolviendo a nadie. Simplemente digo: 'los seres humanos inventamos un órgano para resolver nuestras diferencias y perseguir los delitos y ese órgano que inventó el ser humano en sociedad y en el Estado son los Tribunales de Justicia, ellos son los llamados a imponer la ley en esa materia'. (Francisco Huenchumilla en "Mentiras Verdaderas", 14 de junio de 2014).

Como se observa, el ex intendente logra evadir la carga de la prueba, evitando pronunciarse sobre los posibles culpables en el caso Luchsinger; en este caso, recurriendo a un argumento vinculado a la lógica jurídica, centrándose en el papel que le compete a los Tribunales de Justicia en los causas judiciales, es decir, argumentado desde la autoridad que reviste este poder del Estado.

Estas maniobras estratégicas tienen en consideración el acto perlocutivo del lenguaje, es decir, el efecto del discurso sobre la audiencia; en este caso, evidentemente, las respuestas del ex intendente no se dirigen directamente a los conductores de los programas, en lugar de ello, como político, preocupado por la eficacia, construye su propia audiencia, una audiencia compleja, porque, por una parte, como representante directo de la Presidenta de la República en la región de la Araucanía, debe considerar las expectativas del Gobierno 
sobre su gestión; pero, por otra parte, no es desconocida su filiación étnica como parte del pueblo mapuche, de manera que, en esta disyuntiva, su discurso argumentativo se orienta igualmente a las demandas de las comunidades indígenas. En consecuencia, es posible establecer que su discurso argumentativo está en constante tensión con aquellos presupuestos que ambas audiencias -Estado y pueblo mapuche- esperan de él, a través de sus declaraciones en los medios de comunicación, especialmente en el contexto de su visita al principal sospechoso en el caso Luchsinger, y en relación con los presuntos culpables en este caso judicial.

\subsection{Estructura lógica de esquema argumental de base sintomática, argumento por definición}

En el análisis del discurso de Huenchumilla, se han identificado igualmente esquemas argumentativos de base sintomática por definición (Van Eemeren, Grootendorts y Snoeck Henkemans 2006). En este caso, el ex intendente se refiere al clima sociopolítico en la Araucanía, focalizando el denominado conflicto mapuche:

Tomas Mosciatti: -¿Qué pasa con los testigos protegidos, que algunos cuando se le ha levantado la protección, han sido amenazados? Hay un fiscal que acaba de renunciar, dicen que por amenaza [...] O sea que es una sensación de inseguridad. Y eso sumado a que hay trece fiscales que están con protección personal. ¿Ese es el ambiente que se vive?, o sea, ¿quiénes los amenazan?, bueno... me imagino que serán mapuches, porque son los mapuches los que están siendo investigados por estos fiscales; o sea, fiscales que temen por su vida

Francisco Huenchumilla: -Mire, es que aquí hay una cuestión de fondo que yo la he dicho en innumerables oportunidades, pero la gente parece que no quiere entender exactamente el tema. Yo he dicho que estamos viviendo un conflicto y muchos me dijeron, pero por qué usas la palabra 'conflicto'. Hay un conflicto, porque hay una contraposición de intereses entre el Estado chileno y el pueblo mapuche. He dicho que este es un conflicto grave y que es un conflicto asimétrico. (Francisco Huenchumilla, en Tomás Mosciatti en CNN Chile, 5 junio de 2014)

En esta secuencia discursiva, el ex intendente plantea claramente su punto de vista frente a la existencia del denominado conflicto mapuche, dando a conocer su postura argumental con respecto a la naturaleza de este fenómeno a través de un acto asertivo de afirmación: "Yo he dicho que estamos viviendo un conflicto ... hay un conflicto...". Para defender este punto de vista, utiliza como maniobra estratégica una forma particular de relación sintomática entre el argumento y el punto de vista, en tanto que el primero encierra una conceptualización de la noción de conflicto: "... porque hay una contraposición de intereses entre el Estado chileno y el pueblo mapuche”. Como puede observarse, en la definición proporcionada, el orador se centra en el aspecto dialéctico del término 'conflicto', 
poniendo en evidencia la existencia de dos partes involucradas, a saber, el Estado chileno y el pueblo mapuche, con sus propios intereses y requerimientos. Sin duda, la noción de 'conflicto' planteada por Huenchumilla contrasta con los presupuestos enfatizados por Mosciatti, conductor del programa televisivo, quien al referirse al clima sociopolítico de la Araucanía, focaliza el conflicto solo en una de las partes involucradas, los testigos protegidos y los fiscales: "¿Qué pasa con los testigos protegidos, que algunos cuando se le ha levantado la protección, han sido amenazados? Hay un fiscal que acaba de renunciar, dicen que por amenaza [...] O sea que es una sensación de inseguridad. Y eso sumado a que hay trece fiscales que están con protección personal. ¿Ese es el ambiente que se vive? [...]". A partir de este fragmento discursivo, podría deducirse que los principales afectados serían los testigos protegidos y los fiscales que investigan las causas vinculadas al conflicto mapuche; sin embargo, aun cuando podamos conceder un estilo periodístico específico, como el de Mosciatti, connotado conductor radial y presentador de televisión, resulta de interés observar cómo los medios televisivos reproducen en el discurso, presunciones negativas en torno al denominado conflicto mapuche, enfatizando el fenómeno mediático de criminalización de la protesta indígena, que ha venido advirtiéndose en los últimos años (Mella 2007, Bengoa 2012).

El esquema argumental identificado, puede observarse en la siguiente figura:

Fig. 2. Estructura lógica de esquema argumental de base sintomática, argumento por definición

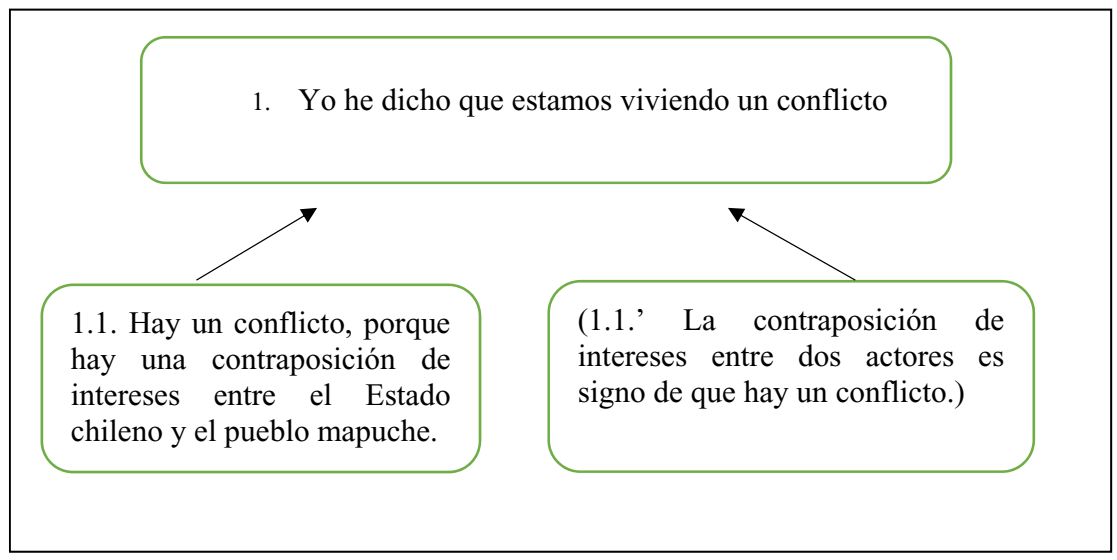

Como puede observarse en la Figura 2, la premisa implícita encierra un tipo de relación de orden semántico entre el argumento y el punto de vista (1.1'), en tanto que el primero muestra una definición específica de la noción de 'conflicto', en términos de una 'contraposición de intereses entre dos actores', que no son otros que el Estado de Chile y el pueblo mapuche (1.1). En este caso, ante la pregunta crítica: ¿hay otros eventos que 
podrian ser fuentes más confiables que tal signo?, se observa la aceptabilidad del argumento al compararse con otras fuentes, ya que, por ejemplo, en la teoría política, la noción de 'conflicto' se define en términos de una oposición, choque o confrontación de dos o más intereses distintos, o también por la contradicción de visiones, voluntades u opiniones (Martínez, 2008). De esta manera, un esquema argumentativo bien fundamentado da cuenta de su razonabilidad y se convierte en una maniobra estratégica, que permite al orador confrontar los presupuestos evocados por Mosciatti en la entrevista estudiada, con respecto al ambiente que se vive en la Araucanía. A su vez, la maniobra sigue el principio de eficacia, ya que, a través del esquema argumentativo, el orador interpela a su audiencia, los principales actores del conflicto: el Estado y el pueblo mapuche. De manera explícita, atribuye al Estado un interés distinto u opuesto a los del pueblo mapuche. La visión particular de Francisco Huenchumilla sobre el conflicto mapuche es reiterada en diversas declaraciones en los medios televisivos, como, por ejemplo, en una intervención como invitado en el programa Mentiras Verdaderas:

Francisco Huenchumilla: -Lo que quiero decir [...] es que hay un desconocimiento respecto de cómo llegó el Estado de Chile ahí. Lo otro es que tenemos un conflicto, yo no lo niego, y yo digo que es un conflicto grave. Más aún yo he empleado la fórmula que este es un conflicto asimétrico. Yo llamo a los entendidos a entender lo que yo estoy diciendo. Es un conflicto asimétrico. Por lo tanto de la más alta gravedad para el Estado. No es un tema que haya inventado yo como Francisco Huenchumilla. Yo estoy prendiendo las luces amarillas, y estoy prendiendo las luces rojas al país.

(Francisco Huenchumilla en "Mentiras Verdaderas", 14 de junio de 2014).

En esta declaración, se observa que la maniobra estratégica del esquema de definición se ve reforzada mediante mecanismos estilísticos específicos como la figura de la repetición aplicada a la noción de 'conflicto'; el uso de expresiones metafóricas en el enunciado 'estoy prendiendo las luces amarillas, y estoy prendiendo las luces rojas al pais', y el recurso léxico de la calificación valorativa, en términos de un 'conflicto' de carácter 'grave' y 'asimétrico', lo que hace presuponer que uno de los actores de este conflicto estaría en clara desventaja respecto del otro. La presencia de recursos estilísticos y retóricos en este discurso contribuyen a la eficacia, pues permitirían captar la atención de la audiencia sobre los hechos presentados (Perelman 1968).

En cualquier caso, el discurso argumentativo de Huenchumilla obedece principalmente al principio de razonabilidad, pues el esquema argumentativo de signos por definición encierra una conceptualización de la noción de 'conflicto' que contrasta con la de otras fuentes, las cuales esgrimen el argumento del 'conflicto mapuche', estigmatizado en los medios tradicionales, asociando el conflicto con un solo actor, el pueblo mapuche, como si fuera este el agente responsable de la situación de confrontación en la región de la Araucanía. 


\subsection{Esquema de argumentación basada en una relación causal}

En el proceso de defensa se utiliza un esquema basado en una relación causal "si el argumento presenta algo como causa del efecto que es mencionado en el punto de vista, como los medios destinados a una finalidad o como una acción con un cierto efecto" (Van Eemeren 2002: 105). De esta forma, en este tipo de razonamiento, se observa cómo un determinado punto de vista se justifica a través de la precisión de un factor causal, en tanto evento o circunstancia que ha determinado una postura o punto de vista particular. Este tipo de estrategia argumentativa se encuentra presente igualmente en el discurso de Francisco Huenchumilla, específicamente con respecto a las políticas del Estado de Chile para enfrentar el denominado conflicto mapuche, develando las limitaciones de la aplicación de la Ley antiterrorista.

\subsubsection{Esquema argumental de base causal}

Francisco Huenchumilla: -Por lo tanto, yo no niego la existencia del conflicto. Lo que yo cuestiono es la forma como el Estado chileno ha enfrentado este conflicto. Llevamos más de veinte años utilizando la Ley Antiterrorista y ¿cuál es el resultado? El resultado es que probablemente vamos a estar condenados por la Corte Interamericana de Derechos Humanos, por aplicar esta Ley, que no resiste ningún estándar internacional en una sociedad democrática.

(Francisco Huenchumilla, en Tomás Mosciatti en CNN Chile, 5 junio de 2014)

En este caso, la argumentación permite plantear un cuestionamiento a las políticas del Estado en relación a un conflicto social de la envergadura que reviste la problemática indígena en Chile. Esta crítica descansa en un argumento causal, referido a la aplicación la Ley antiterrorista, normativa heredada de la Dictadura militar e implementada por el Estado para enfrentar el conflicto en la Araucanía, como mecanismo de represión a las movilizaciones indígenas. Por tanto, en esta secuencia discursiva, se observa la desaprobación explícita a las políticas de los gobiernos de Chile en los últimos veinte años, poniendo en cuestión la efectividad de la Ley Antiterrorista, a la luz de resultados ineficaces en la solución de un conflicto. En su argumentación, el orador utiliza la maniobra causal para enjuiciar el accionar del Estado de Chile, dejando al descubierto el fenómeno de criminalización asociado a las movilizaciones del pueblo mapuche. Lo anterior permite plantear que el denominado conflicto mapuche no se ha enfrentado adecuadamente, debido a que se ha dejado en manos de los Tribunales de Justicia. El esquema argumentativo identificado en el ejemplo se reconstruye en la siguiente figura: 
Fig. 3. Estructura lógica de esquema argumental de base causal

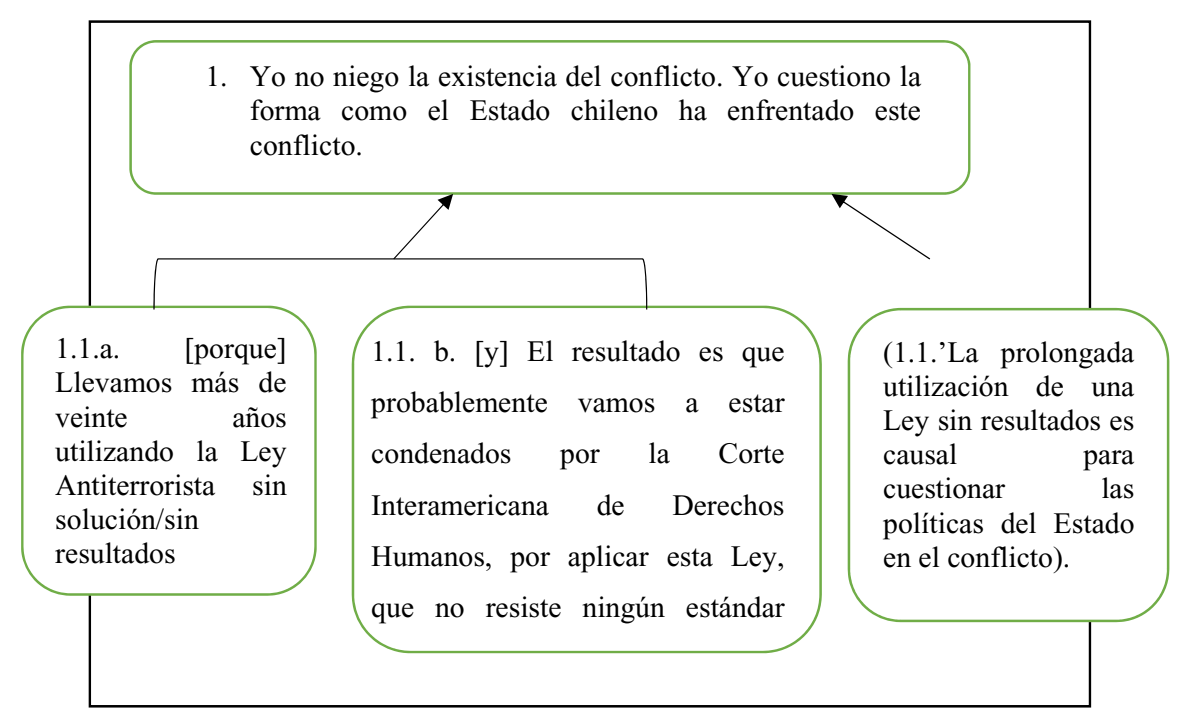

En el análisis de este esquema argumentativo, se puede observar la potencial eficacia del discurso de Huenchumilla a través de dos argumentos coordinados de base causal (1.1.a y 1.1.b). En el primero, se precisa que la aplicación de políticas equivocadas como la Ley Antiterrorista de parte de los distintos Gobiernos no ha dado solución al problema de la Araucanía. El argumento en tanto causa permite justificar y reforzar un cuestionamiento explícito a los distintos Gobiernos, particularmente por la implementación de la mencionada Ley, política incongruente con un sistema de gobierno democrático, tal como se observa en el punto de vista de Huenchumilla: "Por lo tanto, yo no niego la existencia del conflicto. Lo que yo cuestiono es la forma como el Estado chileno ha enfrentado este conflicto." En este caso, cabe destacar, que el primer argumento de base causal se apoya en un segundo argumento, el cual pone énfasis en las consecuencias de las políticas de Gobierno en materia indígena en el plano internacional: "El resultado es que probablemente vamos a estar condenados por la Corte Interamericana de Derechos Humanos, por aplicar esta Ley, que no resiste ningún estándar internacional en una sociedad democrática” (1.1.b.). Este argumento releva la mirada internacional en materia de Derechos Humanos sobre la forma en que el Estado ha enfrentado el conflicto en la Araucanía, aspecto pocas veces mencionado en los medios, considerando la mediatización y criminalización de la protesta indígena. Como puede observarse, el cuestionamiento a la Ley antiterrorista, creada en la dictadura militar, descansa en un argumento causal-pragmático, enfatizando las consecuencias negativas de su aplicación, desde estándares internacionales, particularmente desde los instrumentos de la Corte Interamericana de Derechos Humanos, organismo que 
establece obligaciones para la promoción y protección de los Derechos Humanos en el marco de la Organización de Estados Americanos (OEA), de la cual Chile es miembro. Desde el punto analítico, ¿̨hay realmente correlación entre argumento y punto de vista? Esta pregunta crítica puede responderse a través de la premisa implícita reconstruida, la cual pone en evidencia la relación entre la causa, como razón suficiente para adoptar una postura de cuestionamiento a las políticas de Estado en el tema del conflicto mapuche, estableciéndose en el discurso que la prolongada utilización de una Ley sin resultados es causal para cuestionar las politicas del Estado en el conflicto (1.1.'). En la misma línea, como pregunta crítica, ¿hay alguna razón para pensar que la correlación es más que una simple coincidencia? En este caso, la maniobra estratégica se refiere a causales relevantes para cuestionar las políticas del Estado de Chile hacia los pueblos indígenas, vale decir, no es una suerte del azar que la Ley Antiterrorista, en lugar de dar solución al conflicto, pareciera intensificarlo, y prolongarlo en el tiempo. De hecho, la aplicación de la Ley Antiterrorista ha recibido serios reparos de parte de diferentes entidades y organismos internacionales, que han enfatizado que debe revisarse este instrumento jurídico, por vulnerar el derecho a la presunción de inocencia de los acusados. De manera más precisa, se cuestiona la figura de los testigos protegidos, la prisión preventiva de los imputados por actos terroristas y otras restricciones al debido proceso (Aylwin 2010). Estos argumentos también son recogidos por el ex intendente en muchas de sus declaraciones, como puede apreciarse en una de sus intervenciones en el programa Mentiras Verdaderas:

Conductor: La ley antiterrorista permite irregularidades en la fiscalía.

F. Huenchumilla: No, permite, por ejemplo, la tenencia de testigos protegidos, pero más que protegidos testigos encapuchados, testigos anónimos, testigos que tú no los puedes conocer, que la defensa no tiene derecho a saber quiénes son. Entonces eso se presta para cualquier cosa, entonces, mire, la Ley Antiterrorista no es la solución, si hubiera sido la solución, basta haber aplicado todo estos años la Ley Antiterrorista, el problema se hubiera solucionado, no se ha solucionado mediante esas medidas, porque el problema es de otra naturaleza.

(Francisco Huenchumilla en "Mentiras Verdaderas”, 14 de junio de 2014).

Asimismo, tras los cuestionamientos a la Ley antiterrorista emerge un nuevo tópico de controversia en el discurso de Huenchumilla, en relación con el planteamiento de un diagnóstico sobre la situación en la Araucanía. En muchas de sus declaraciones, el ex intendente señala categóricamente que los gobiernos "han equivocado el diagnóstico", pretendiendo que la vía judicial y policial son las soluciones para el problema en la región (Cayuqueo, 2015). En cuanto a la secuencia temática concerniente a un diagnóstico sobre la situación en la Araucanía, encontramos maniobras estratégicas de base causal en el discurso de Huenchumilla, como se describe a continuación. 


\subsubsection{Esquema argumental de base causal}

Mónica G.: ¿Cómo atacas la violencia entonces?, porque no vamos a decir que la situación había mejorado, ¿cuál es el origen de esa violencia?, ¿cómo se ataca si no es policial?

Francisco H.: Bueno, mira, yo lo que digo, es que para atacar toda esta violencia que ha existido acá en estos 20 años con todos estos hechos, los gobiernos han usado todos los instrumentos que hay, se han querellado por la Ley de Seguridad Interior del Estado, por el Código de Justicia Militar, por el Código Penal con los delitos que están ahí, por la Ley de Control de Armas, por la Ley Antiterrorista; se han usado todas las posibilidades posibles, ¿y cuáles han sido el resultado?, el cero; estamos en lo mismo, después de dieciocho años, entonces, ¿qué digo yo?, que lo que hay que hacer ahí es tomar medidas políticas respecto del fenómeno que tenemos ahí en la región de la Araucanía.

(Francisco Huenchumilla en Tolerancia Cero, 30 de agosto de 2015).

Sin duda, el tema de la violencia en la Araucanía es una de las aristas más álgidas en relación con el denominado conflicto mapuche, y con la protesta indígena en la región. El ex intendente plantea su visión al respecto, refutando que la solución sea la vía policial o las de los tribunales de justicia. Esto queda de manifiesto de manera explícita en varias de sus declaraciones, tal como se observa en la secuencia presentada: “... estamos en lo mismo, después de dieciocho años, entonces, ¿qué digo yo?, que lo que hay que hacer ahi es tomar medidas politicas respecto del fenómeno que tenemos ahi en la región de la Araucanía".

El esquema argumentativo de base causal, identificado en esta secuencia discursiva se muestra en la siguiente figura.

Como vemos en este último caso, el punto de vista de Huenchumilla es que la solución al conflicto en la Araucanía es de orden político, basándose en un esquema argumentativo de lógica causa-efecto. La principal causa para proponer una nueva forma de enfrentar el conflicto obedece a los fracasos derivados der la vía judicial y policial, como se muestra en la reconstrucción de la premisa implícita (1.1'): El fracaso de medidas policiales y juridicas es causal para adoptar soluciones politicas en la región de la Araucanía. En otras declaraciones, Francisco Huenchumilla critica igualmente las políticas de los gobiernos en los últimos veinte años, enfatizando que el problema no es de orden público, sino de naturaleza política, como se observa en la siguiente secuencia:

Francisco Huenchumilla: - ... bueno la responsabilidad digo yo es del Estado, yo coincido en eso... pero sobre todo hablemos de los últimos veinte ańos, porque se ha pensado que siempre el problema de ahí es un problema de pobreza y un problema de orden público. Por lo tanto, se han hecho políticas públicas para combatir la extrema pobreza con bastante malos resultados y se han hecho políticas 
Fig. 4. Estructura lógica de esquema argumental de base causal

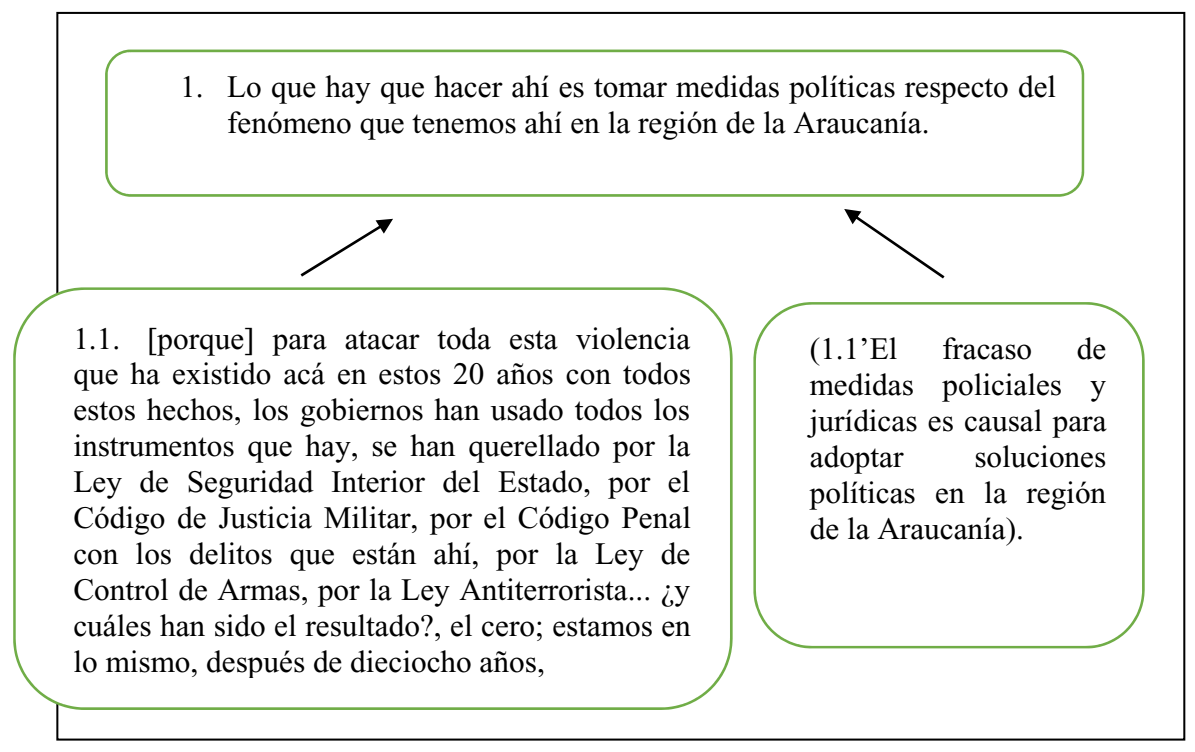

públicas con malos resultados también, porque seguimos exactamente igual. Yo el cambio de giro que hago que no es un problema solo de pobreza, sino que es un problema político y entonces yo abro este enfermo y digo con qué me encuentro, que hace 130 años atrás los mapuches tenían 5 millones de hectáreas y el Estado les quita 4 millones y medio y lo reduce a 500 mil.

(Francisco Huenchumilla en "Mentiras Verdaderas", 14 de junio de 2014)

El planteamiento de un cambio de giro para solucionar el conflicto en la Araucanía, a través de la vía política, constituye un nuevo discurso en el debate sobre las políticas en materia indígena en Chile, sentando un nuevo precedente en la forma de abordar el movimiento indígena en los medios. En otras declaraciones, el ex intendente subraya la necesidad de reconocer que estamos frente a un problema de naturaleza política, lo que implica al Estado aceptar el reconocimiento de la existencia y la naturaleza del actor, el pueblo mapuche, que lo interpela mediante la existencia de este conflicto (Cayuqueo 2015). Como hemos observado en el análisis, el discurso de Huenchumilla logra posicionar los asuntos indígenas y el denominado conflicto mapuche, desde una nueva mirada, que va más allá de aspectos judiciales, o policiales, los cuales han resultado el principal foco abordado por los medios de comunicación en los últimos años. Frente a este panorama, el punto de vista del ex intendente basado en un diagnóstico sobre el fracaso de las medidas de orden judicial, policial y público, se anticipa hacia una 
propuesta política concreta para la solución de un innegable conflicto en la región, ${ }^{8}$ la cual no alcanza a presentar al gobierno, debido a circunstancias políticas que terminan con su gestión y que, de alguna manera, gatillan su controversial salida del cargo de intendente de La Araucanía, por distintas presiones en la región, como las del gremio de los camioneros. En definitiva, los principios de razonabilidad y eficacia están presentes en el discurso del ex intendente, plasmados en la expresión de sus puntos de vista y en las maniobras estratégicas. La razonabilidad queda de manifiesto en el uso de esquema argumentativos, en general en congruencia con las máximas griceanas, y con las preguntas críticas aplicadas a un discurso razonable. La eficacia se observa en las consideraciones retóricas, que apuntan tanto al uso de maniobras estratégicas adecuadas al contexto, como al alcance de la argumentación sobre distintas audiencias, lo cual se observa, entre otros, a través de un estilo retórico directo, en cuanto a su posicionamiento sobre el conflicto mapuche, claramente graficado en las propias palabras de Francisco Huenchumilla en Tolerencia Cero, y en declaraciones posteriores a su gestión: "El gobierno le tuvo miedo a mi propuesta".

\section{Conclusiones}

En el análisis del discurso de Francisco Huenchumilla en torno al denominado conflicto mapuche, hemos encontrado puntos de vista y esquemas argumentativos, que permiten al ex intendente posicionar la relevancia del problema de la situación de conflicto en la Araucanía en los medios. Los principales puntos de vista dan cuenta de la postura del ex intendente frente a los problemas en la Araucanía, relevando especialmente el conflicto entre Estado y pueblo mapuche como un problema político, cuya solución no es la vía judicial ni la policial. Entre sus argumentos esgrime como maniobra estratégica el fracaso de la Ley Antiterrorista, como argumento causal.

Desde la maniobra estratégica, se observa la tensión entre el principio de eficacia y el de razonabilidad en su discurso, especialmente en relación con su postura frente al caso Luchsinger, donde el ex intendente se muestra como un político mesurado, que no se pronuncia ni responde directamente las preguntas en la interacción televisiva. Desde esta perspectiva, emerge la importancia que tiene para el ex intendente como político, la eficacia, centrada en las expectativas de sus audiencias; en este caso, el Gobierno y el pueblo mapuche.

Los principales esquemas identificados en el discurso del ex intendente son de base sintomática y causal, los cuales permiten reforzar claramente sus puntos de vista. Destaca el tipo de argumento por definición para plantear su postura sobre la existencia de un conflicto real en la Araucanía, puntualizando la contraposición de intereses entre dos

\footnotetext{
${ }^{8}$ La propuesta de Huenchumilla que no alcanzó a presentar al gobierno consta de 48 páginas y se basa en dos ejes principales: Chile como Estado plurinacional y devolución de tierras al pueblo mapuche (Cayuqueo 2015, Huenchumilla 2017).
} 
partes y precisando a los principales actores involucrados: el Estado chileno y el pueblo mapuche. Este hallazgo es interesante de relevar, ya que es común que el conflicto se asocie discursivamente con un único actor -el pueblo mapuche-, focalizando la responsabilidad del conflicto en una sola parte de la discusión, lo que resulta una incongruencia desde la definición del término.

Desde un análisis argumentativo del discurso aplicado a las interacciones estudiadas, la descripción de los hechos en torno al denominado conflicto mapuche, específicamente en las preguntas realizadas por los presentadores o conductores de los programas televisivos, da cuenta de la 'criminalización' del conflicto a nivel discursivo en los medios, pues la protesta indígena en la Araucanía se presenta ante la opinión pública como una verdadera pendiente resbaladiza, como si las reales víctimas de la situación en la Araucanía fueran los fiscales que investigan los hechos, el Ministerio| público o el Estado. La interrogantes es clara: ¿En qué momento de la historia de este conflicto, los medios, el Estado o la sociedad en su conjunto reconocerá al pueblo mapuche como actor y, a la vez, como víctima de este conflicto?

Por límites de espacio, no hemos analizado de manera exhaustiva el triángulo de la maniobra estratégica, dejando de lado aspectos retóricos y estilísticos que contribuyen a la fuerza argumentativa de un discurso eficaz. Sin embargo, ningún tipo de audiencia podría pasar por alto la eficacia del discurso de Huenchumilla, ya sea por el contenido proposicional de sus argumentos, como por la fuerza argumentativa de estos, que van desde la referencia a la historia de Chile, a la génesis del conflicto en la Araucanía, a sus causales, así como al Derecho Internacional, proyectando finalmente una propuesta de solución al conflicto en la Araucanía. Estos hallazgos merecen evidentemente un estudio más acabado desde la maniobra estratégica, por tratarse de un discurso político que sienta un precedente en la forma de abordar el conflicto entre el Estado y el pueblo mapuche, posicionándolo como un problema de índole política en los medios, mediante un discurso directo, complejo y concluyente, en virtud de la variedad y pertinencia de los esquemas argumentativos utilizados.

\section{Obras CiTADAS}

Austin, John, ed. 1982. Cómo hacer cosas con palabras: Palabras y acciones. Trad. Genaro Carrió y Eduardo Rabossi. Barcelona: Paidós.

Amolef, Fresia. s.f. La alteridad en el discurso mediático. Los Mapuches y la prensa chilena. Web. 27 de dic. 2017.

Aylwin, José. 2010. La aplicación de la ley $N^{\circ} 18.314$ que "determina conductas terroristas y fija su penalidad" a las causas que involucran a integrantes del pueblo mapuche por hechos relacionados con sus demandas por tierras y sus implicancias desde la perspectiva de los derechos humanos. Web.

Bengoa, José. 2012. Mapuche: Procesos, politicas y culturas en el Chile del Bicentenario. Santiago de Chile: Catalonia Ltda. 
Bengoa, José y Natalia Caniguan. 2012. “Los Mapuche y el Bicentenario”. Mapuche: Procesos, politicas y culturas en el Chile del Bicentenario. Santiago de Chile: Catalonia Ltda.

Camps, Anna y Joaquín Dolz. 1995. "Introducción: Enseñar a argumentar: un desafío para la escuela actual”. Comunicación, Lenguaje y Educación. 5-8. Web. 04 de ene. de 2018. Cayuqueo, Pedro. 2015. Huenchumilla. La historia del hombre de oro. Santiago de Chile: Catalonia.

Durán, Teresa. 2007. "Diversidad Cultural y Derechos Humanos”. Patrimonio Educacional Mapuche Vol. II. Temuco: Coordinación Editorial UC. 159-174. Gille, Johan. 2001. Pautas argumentativas en el diálogo espontáneo Un estudio de conversaciones intra e interculturales. Tesis Doctoral Inédita. Estocolmo: Stockholm University.

Goivocic, Igor. 2015. "Campos conceptuales, perspectivas de análisis y ciclos históricos en el estudio del movimiento mapuche (1870-1990)". Conflictos étnicos, sociales y económicos Araucanía 1900-2014. Santiago: Pehuén. 19-44.

Grice, Herbert. 1975. "Logic and conversation". Syntax and semantics 3: Speech arts. Cambridge: Academic Pr. 41-58. Web.

Huenchumilla, Francisco. 2017. Plurinacionalidad. El nuevo Pacto. Santiago: Pehuén Editores.

Levinson, Stephen. 1983. Pragmatics. Cambridege: Cambridge University Press. Mariafioti, Roberto, Cristian Santibáñez. 2010. Teoría de la argumentación. A 50 años de Perelman y Toulmin. Buenos Aires: Editorial Biblos.

Martínez, María Cristina. 2005. La construcción del proceso argumentativo en el discurso. Perspectivas teóricas y trabajos prácticos. Cátedra UNESCO. Cali: Universidad del Valle. Martínez, Miguel. 2008. "Elementos para una teoría general del conflicto”. Heterotopía 39: 47-74.

Mella, Eduardo. 2007. Los mapuche ante la justicia. La criminalización de la protesta indígena en Chile. Santiago: LOM Ediciones.

Pairicán, Fernando. 2015. "Weuwaiñ. 2: la invención de la tradición en la rebelión del movimiento mapuche (1990-2010)". Conflictos étnicos, sociales y económicos. Araucanía 1900-2014. Santiago: Pehuén. 187- 214.

Perelman, Chaïm. 1968. Éléments d'une théorie de l'argumentation. Bruselas: Prensas Universitarias de Bruselas.

Perelman, Chaïm \& Lucie Olbrechts-Tyteca. 2009. Tratado de la argumentación. Trad. Julia Sevilla. Madrid: Gredos.

Pinto, Jorge. 2015. Conflictos étnicos, sociales y económicos. Araucanía 1900-2014. Santiago: Pehuén.

Rodríguez, Simone, Carlos F. Domínguez. 2011. «Sociedades plurales, multiculturalismo y derechos indígenas en América Latina». Politica y Cultura 35: 49-66. Schegloff,EmanuelyHarveySacks. 1973. “Openingupclosings”. Semiotica7,4:289-327. Searle, John. 1969. Speech Acts. Cambridge: Cambridge University Press. Toulmin, Stephen. 1958. The Uses of Argument. Cambridge: Cambridge U. Press. 
Toulmin, Stephen, Richard Rieke y Allan Janik 1984. An Introduction to Reasoning. Nueva York: MacMillan.

Tusón, Amparo. 1997. Análisis de la conversación. Barcelona: Editorial Ariel.

Van Eemeren, Frans, Rob Grootendorst. ed. 2002. Argumentación, comunicación y falacias. Una perspectiva pragma-dialéctica. Trad. Celso López y Ana María Vicuña. Santiago: Ed. Universidad Católica de Chile. . 2004. A Systematic Theory of Argumentation. The Pragma-Dialectical Approach. Cambridge: Cambridge University Press.

Van Eemeren, Frans, Rob Grootendorst y Francisca Snoeck Henkemans. 2006. Argumentación. Análisis, evaluación, presentación. Trad. Roberto Marafioti. Buenos Aires: Editorial Biblos.

Van Eemeren, Frans, Peter Houtlosser, ed. 2007. "Maniobrando estratégicamente: Manteniendo un delicado balance". Trad. Cristian Santibáńez. Estudios en argumentación y retórica. Teorías contemporáneas y aplicaciones. Concepción: Ed. Universidad de Concepción. 83-121.

Van Eemeren, Frans, ed. 2012. Maniobras estratégicas en el discurso argumentativo. Trad. Cristian Santibánez. Madrid: Plaza y Valdés Editores.

Vergara, Jorge, Hans Gundermann y Rolf Foerster. 2013. Estado, Conflicto étnico y Cultura. Estudio sobre pueblos indigenas en Chile. Chile: Ocho Libros.

Walton, Douglas. 1989. Informal logic. A handbook for critical argumentation. Cambridge: University Press.

Yañez, N. 2007. Prólogo. Los mapuche ante la justicia. La criminalización de la protesta indígena en Chile. Por Eduardo Mella. Santiago: LOM Ediciones. 11-16. 
